MATHEMATICS OF COMPUTATION

Volume 73 , Number 246 , Pages $813-825$

S 0025-5718(03)01564-3

Article electronically published on August 19, 2003

\title{
A MONTE CARLO ALGORITHM FOR WEIGHTED INTEGRATION OVER $\mathbb{R}^{d}$
}

\author{
PIOTR GAJDA, YOUMING LI, LESZEK PLASKOTA, \\ AND GRZEGORZ W. WASILKOWSKI
}

\begin{abstract}
We present and analyze a new randomized algorithm for numerical computation of weighted integrals over the unbounded domain $\mathbb{R}^{d}$. The algorithm and its desirable theoretical properties are derived based on certain stochastic assumptions about the integrands. It is easy to implement, enjoys $O\left(n^{-1 / 2}\right)$ convergence rate, and uses only standard random number generators. Numerical results are also included.
\end{abstract}

\section{INTRODUCTION}

Numerical multiple integration has been extensively studied and there is a huge volume of theoretical results as well as algorithms in case the integration domain $D$ is bounded, say $D=[0,1]^{d}$ is the unit cube. Much less is known for integration over unbounded domains such as $D=\mathbb{R}^{d}$. This is in spite of the fact that weighted integrals of the form

$$
I_{\rho}(f)=\int_{\mathbb{R}^{d}} f(\mathbf{x}) \rho(\mathbf{x}) d \mathbf{x}
$$

appear in a number of important applications including mathematical finance, physics, and scientific computing.

The unbounded domain $D$ adds some extra layers of difficulty to the integration problem, as compared to $D=[0,1]^{d}$. We now list a few of them.

1. Algorithms that are efficient for unweighted integration over $[0,1]^{d}$ can be formally used, by a change of variables, for weighted integration over $\mathbb{R}^{d}$. However, such a procedure transforms, as a rule, a class of smooth functions to a class of functions with singularities, which makes the theoretical results for $[0,1]^{d}$ not applicable for $\mathbb{R}^{d}$. One could also use a truncation of the domain to a bounded cube, but then the size of the resulting domain has to very much depend on the specific integrand which makes it difficult to obtain a general purpose algorithm.

2. Uniform distribution on $D$, that is commonly utilized in Monte Carlo algorithms for $D=[0,1]^{d}$, does not exist for $D=\mathbb{R}^{d}$. Hence, the question arises of how to select random samples from $\mathbb{R}^{d}$. One could obviously select $\mathbf{x}^{1}, \ldots, \mathbf{x}^{n}$ according to density proportional to the weight $\rho$. However, for the errors to be at

Received by the editor February 18, 2002 and, in revised form, July 23, 2002.

2000 Mathematics Subject Classification. Primary 65D30, 65C05.

Key words and phrases. Numerical multiple integration, Monte Carlo methods, average case error. 
least proportional to $n^{-1 / 2}$, one has to assume that

$$
\int_{\mathbb{R}^{d}} f^{2}(\mathbf{x}) \rho(\mathbf{x}) d \mathbf{x}<\infty
$$

which need not be the case and actually is not needed (see explanation below). Moreover, for complicated weights $\rho$, generating the corresponding i.i.d. samples $\mathbf{x}^{i}$ could be very difficult and expensive.

3. There are few very good (deterministic or randomized) algorithms for unbounded domains, such as stochastic spherical-radial rules or algorithms using lowdiscrepancy sequences; see, e.g., [1, 6, 9, 11, 12. However, they either work for specific weights $\rho$ like, e.g., Gaussian weight, and for specific integrands like those describing the value of mortgage-backed securities, or they require complicated random number generators and have a significant overhead cost. For some of these methods, there are no theoretical error bounds known. For other methods only $a$ posteriori error bounds are available since, due to their complication, any a priori error bounds for large classes of integrands are extremely difficult to obtain.

The aim of the present paper is to propose a randomized algorithm that is free of the problems mentioned above, that is, an algorithm that is in a way universal: very easy to implement, requires only uniform random number generator, has desirable theoretical properties for a large class of weight functions $\rho$ and a large class of integrands $f$.

For that end, we made certain stochastic assumptions on the integrands $f$ that allow us to analyze average case errors (as opposed to the worst case errors) $\sqrt{1}$ of the algorithm. We stress that these stochastic assumptions served only to guide us in developing the algorithm, and we believe that it could/should be used in a much more general framework. Indeed, to maximize its potential applicability, we have chosen a relatively nonrestrictive assumption (only Hölder continuity). Moreover, as can also be shown, the error of our algorithm converges to zero with $n$ for any (a.e.) continuous $f$ with finite $I_{\rho}(f)$.

More specifically, we consider approximating integrals $I_{\rho}$ of the form (1) for a given (but arbitrary) weight $\rho$ of the isotropic form,

$$
\rho(\mathbf{x})=\omega(\|\mathbf{x}\|) \text {. }
$$

Here and elsewhere $\|\cdot\|$ denotes the Euclidean norm. The space of integrands $f$ is equipped with a Gaussian measure or, equivalently, $f$ is a Gaussian random process, so that average case errors of algorithms and average case complexity of the problem can be investigated. The average case complexity of this problem has recently been studied in [13] and the current paper can be viewed as a continuation of that publication. Among others, the following is shown in 13. Let the process $f$ be $r$-times differentiable, and let the $r$ th derivative be Hölder continuous (in the mean square sense) with the exponent $\beta$. Then there exists an algorithm using $n$ values of $f$ whose expected error does not exceed $n^{-1 / 2-(r+\beta) / d}$. A special randomized algorithm that achieves this rate of convergence has been exhibited there. However, the algorithm requires random points drawn from a distribution that highly depends on the weight function $\rho$ and the covariance of the process $f$. Moreover, the combinatorial cost (i.e., the number of arithmetic operations) of the algorithm is exponential in $d$, the number of variables.

\footnotetext{
${ }^{1}$ Actually, our classes of $\rho$ and $f$ are large so that the worst case errors are unbounded.
} 
In this paper, we assume that $f$ is an isotropic fractional Wiener process with the parameter $0<\beta \leq 1 / 2$. This means, in particular, $r=0$, i.e., no differentiability is assumed. We also assume that

$$
\int_{\mathbb{R}^{d}}\|\mathbf{x}\|^{\beta} \rho(\mathbf{x}) d \mathbf{x}<\infty
$$

since this is a necessary and sufficient condition for $I_{\rho}(f)$ to be well defined (with probability one); see [13]. Then our randomized (Monte Carlo) algorithm denoted by $\mathcal{Q}_{n}^{*}$ has the average error satisfying

$$
\limsup _{n \rightarrow \infty} \sqrt{n} \cdot \operatorname{error}\left(\mathcal{Q}_{n}^{*}\right) \leq \int_{\mathbb{R}^{d}}\|\mathbf{x}\|^{\beta} \rho(\mathbf{x}) d \mathbf{x} .
$$

This error bound is greater than the error bound from [13] by a factor proportional to $n^{\beta / d}$; however, the difference is negligible for large $d$.

The algorithm uses the well-known idea of stratified sampling (see, e.g., 7 8); however, it is now applied to the unbounded domain $\mathbb{R}^{d}$. That is, the domain $\mathbb{R}^{d}$ is divided into a finite number of rings $P_{i}$ centered at zero with appropriately chosen radii (the last ring is unbounded). From each (except the last) ring $P_{i}$, a special number $n_{i}$ is computed. Next, $n_{i}$ i.i.d. points are randomly chosen from the uniform distribution on $P_{i}$. Such points can easily be generated at cost proportional to $d \cdot n$ by using a uniform random number generator; see, e.g., [3, [5].

We now comment on the assumption (4). As we already mentioned, it is necessary for the integrals $I_{\rho}(f)$ to be well defined. Clearly, it is less restrictive than $\int_{\mathbb{R}^{d}}\|\mathbf{x}\|^{2 \beta} \rho(\mathbf{x}) d \mathbf{x}<\infty$ since there are weights $\rho$ satisfying (4) with infinite integral of $\|\mathbf{x}\|^{2 \beta} \rho(\mathbf{x})$. Note, however, that the latter integral is the expected value (with respect to $f$ ) of the integral of $f^{2} \cdot \rho$,

$$
\mathbb{E}\left(\int_{\mathbb{R}^{d}} f^{2}(\mathbf{x}) \rho(\mathbf{x}) d \mathbf{x}\right)=\int_{\mathbb{R}^{d}}\|\mathbf{x}\|^{2 \beta} \rho(\mathbf{x}) d \mathbf{x} .
$$

This means, in particular, that our algorithm preserves its convergence even if (2) does not hold.

The algorithm was implemented and tested for different dimensions $d$, weights $\rho$, and deterministically chosen functions $f$. Among others, we tested the computation of the mortgage backed securities. This is a problem for which a number of very specialized algorithms have recently been designed and tested; see, e.g., 2, 12. It is noticeable that the errors of our algorithm are comparable to that obtained by the quasi-Monte Carlo method in [12] even though, unlike the specialized algorithms, our method was designed and works for a large class of integrands and weights $\rho$.

\section{The ALGORITHM}

We consider the following Monte Carlo algorithm for approximating the integral (11).

The space $\mathbb{R}^{d}$ is divided into rings

$$
P_{i}=\left\{\mathbf{x} \in \mathbb{R}^{d}: r_{i-1} \leq\|\mathbf{x}\|<r_{i}\right\}, \quad i \geq 1,
$$

where $0=r_{0}<r_{1}<r_{2}<\cdots$ is an infinite sequence with $\lim _{i \rightarrow \infty} r_{i}=\infty$. Given a positive integer $n$, nonnegative integers $n_{i}$ are selected such that

$$
n=\sum_{i=1}^{\infty} n_{i}<\infty
$$


This implies, in particular, that $n_{i}>0$ for only finitely many indices $i$. For positive $n_{i}$, the integrals

$$
\operatorname{Int}_{\rho}^{i}(f):=\int_{P_{i}} f(\mathbf{x}) \rho(\mathbf{x}) d \mathbf{x}
$$

are approximated by

$$
\mathcal{Q}_{n_{i}}^{i}(f)=\frac{\operatorname{Vol}\left(P_{i}\right)}{n_{i}} \cdot \sum_{s=1}^{n_{i}} f\left(\mathbf{t}_{i, s}\right) \cdot \rho\left(\mathbf{t}_{i, s}\right),
$$

where the points $\mathbf{t}_{i, s} \in P_{i}$ are chosen randomly and independently according to the uniform distribution on $P_{i}$. For $n_{i}=0$, we set $\mathcal{Q}_{0}^{i}(f)=0$. The approximation to $\operatorname{Int}_{\rho}(f)$ is then given as

$$
\mathcal{Q}_{n}(f)=\sum_{i=1}^{\infty} \mathcal{Q}_{n_{i}}^{i}(f)
$$

Note that $\mathcal{Q}_{n}$ is uniquely determined by the choice of the parameters $r_{i}$ and $n_{i}$ for $i \geq 1$.

Theoretical properties of the algorithm $\mathcal{Q}_{n}$ will be derived based on the assumption that the integrand $f: \mathbb{R}^{d} \rightarrow \mathbb{R}$ is the isotropic fractional Wiener process with parameter $0<\beta \leq 1 / 2$. That is, $f$ is the zero mean Gaussian process with covariance kernel

$$
K(\mathbf{x}, \mathbf{y})=\mathbb{E}(f(\mathbf{x}) f(\mathbf{y}))=\frac{1}{2}\left(\|\mathbf{x}\|^{2 \beta}+\|\mathbf{y}\|^{2 \beta}-\|\mathbf{x}-\mathbf{y}\|^{2 \beta}\right) .
$$

Then, for the integration problem (1) to be well defined, we have to assume that

$$
\int_{\mathbb{R}^{d}} \sqrt{K(\mathbf{x}, \mathbf{x})} \cdot \rho(\mathbf{x}) d \mathbf{x}=\int_{\mathbb{R}^{d}}\|x\|^{\beta} \cdot \rho(\mathbf{x}) d \mathbf{x}<\infty .
$$

Indeed, (5) is a necessary and sufficient condition for $\operatorname{Int}_{\rho}(f)$ to exist and be finite with probability one; see [13]. In the setting of this paper, (5) is equivalent to

$$
\int_{0}^{\infty} t^{d-1+\beta} \omega(t) d t<\infty
$$

which follows from the fact that for $t=\|x\|$ we have $d \mathbf{x}=d c_{d} t^{d-1} d t$ with

$$
c_{d}=\frac{\pi^{d / 2}}{\Gamma(d / 2+1)} .
$$

We also make some specific assumptions on $\omega$ in (3); namely,

- $\omega$ is Riemann integrable on any finite interval,

- there is $T>0$ such that $\omega$ is monotonically nonincreasing on $[T, \infty)$.

See [14] where the necessity of these assumptions is discussed.

We are interested in the expected error

$$
\operatorname{error}\left(\mathcal{Q}_{n}\right)=\left(\mathbb{E}\left(\operatorname{Int}_{\rho}(f)-\mathcal{Q}_{n}(f)\right)^{2}\right)^{1 / 2},
$$

where the expectation is taken with respect to $f$ and the points $\mathbf{t}_{i, s}$.

Our main result reads as follows. 
Theorem 1. For any $n \geq 1$, there exists a choice of the parameters $r_{i}$ and $n_{i}$ such that for the resulting algorithm $\mathcal{Q}_{n}^{*}$ we have

$$
\begin{aligned}
\limsup _{n \rightarrow \infty} \sqrt{n} \cdot \operatorname{error}\left(\mathcal{Q}_{n}^{*}\right) & \leq \int_{\mathbb{R}^{d}}\|x\|^{\beta} \cdot \rho(\mathbf{x}) d \mathbf{x} \\
& =d \cdot c_{d} \cdot \int_{0}^{\infty} t^{d-1+\beta} \cdot \omega(t) d t .
\end{aligned}
$$

\section{Proof}

In the definition and analysis of the error of $\mathcal{Q}_{n}^{*}$ we make use of the ideas from the proof of Theorems 1 and 4 in [14], where deterministic algorithms in case $d=1$ are considered.

That is, we first choose $M>T$ and integer $m \geq 1$. The radii $r_{i}$ of the rings $P_{i}$ are defined as

$$
r_{i}= \begin{cases}i \cdot M / m & \text { for } \quad 0 \leq i \leq m, \\ M \cdot 2^{i-m} & \text { for } i \geq m+1 .\end{cases}
$$

The choice of $n_{i}$ 's depends on two other nonnegative integers $k_{L}$ and $k_{R}$ as follows. Let

$$
a_{i}=\operatorname{Vol}\left(P_{i}\right) \cdot r_{i}^{\beta} \cdot \omega_{i} \quad \text { with } \omega_{i}=\sup _{r_{i-1} \leq t \leq r_{i}} \omega(t), \quad i \geq 1
$$

We immediately make two important observations. First, by isotropicity of $\rho$ and $\mathbf{x} \mapsto K(\mathbf{x}, \mathbf{x})=\|x\|^{2 \beta}$ and by Riemann integrability of $\omega$, we have

$$
\lim _{m \rightarrow \infty} \sum_{i=1}^{m} a_{i}=\int_{\|\mathbf{x}\| \leq M}\|x\|^{\beta} \cdot \rho(\mathbf{x}) d \mathbf{x} .
$$

Second, since $\operatorname{Vol}\left(P_{i}\right)=2^{d} \operatorname{Vol}\left(P_{i-1}\right), r_{i}=2 r_{i-1}$, and $\omega_{i}=\omega\left(r_{i-1}\right)$ for $i \geq m+1$,

$$
\begin{aligned}
\int_{\|\mathbf{x}\| \geq M}\|x\|^{\beta} \rho(\mathbf{x}) d \mathbf{x} & \leq \sum_{i=m+1}^{\infty} a_{i} \\
& \leq 2^{d+1} \cdot \int_{\|\mathbf{x}\| \geq M}\|x\|^{\beta} \rho(\mathbf{x}) d \mathbf{x}+a_{m+1} .
\end{aligned}
$$

This implies, in particular, that $\sum_{i=m+1}^{\infty} a_{i}<\infty$.

For simplicity of presentation, we assume that $\left\{a_{i}\right\}_{i \geq m+1}$ is a nonincreasing sequence. This is without any loss of generality since otherwise we would only need to renumber the sequence. For $1 \leq i \leq m$ we set

$$
n_{i}=\left\lceil\frac{a_{i}}{\sum_{s=1}^{m} a_{s}} \cdot k_{L}\right\rceil,
$$

for $m+1 \leq i \leq m+k_{R}$ we set

$$
n_{i}=\left\lceil\frac{a_{i}}{\sum_{s=m+1}^{m+k_{R}} a_{s}} \cdot k_{R}\right\rceil,
$$

and $n_{i}=0$ for $i \geq m+k_{R}+1$.

It is easy to observe that the method just described uses altogether

$$
n=\sum_{i=1}^{m} n_{i}+\sum_{i=m+1}^{m+k_{R}} n_{i} \leq m+k_{L}+2 \cdot k_{R}
$$

random points. 
We now pass to the error analysis. Since for $i$ with $n_{i} \geq 1$ the random variables

$$
\int_{P_{i}} f(\mathbf{x}) \rho(\mathbf{x}) d \mathbf{x}-\mathcal{Q}_{n_{i}}^{i}(f)
$$

are pairwise independent, we have

$$
\mathbb{E}\left(\int_{\|x\| \leq M} f(\mathbf{x}) \rho(\mathbf{x}) d \mathbf{x}-\sum_{i=1}^{m} \mathcal{Q}_{n_{i}}^{i}(f)\right)^{2}=\sum_{i=1}^{m} \mathbb{E}\left(\operatorname{Int}_{\rho}^{i}(f)-\mathcal{Q}_{n_{i}}^{i}(f)\right)^{2} .
$$

By standard calculations,

$$
\mathbb{E}\left(\operatorname{Int}_{\rho}^{i}(f)-\mathcal{Q}_{n_{i}}^{i}(f)\right)^{2} \leq \frac{\operatorname{Vol}\left(P_{i}\right)}{n_{i}} \int_{P_{i}} K(\mathbf{x}, \mathbf{x}) \rho^{2}(\mathbf{x}) d \mathbf{x} \leq \frac{a_{i}^{2}}{n_{i}}
$$

Hence, using (8),

$$
\mathbb{E}\left(\int_{\|x\| \leq M} f(\mathbf{x}) \rho(\mathbf{x}) d \mathbf{x}-\sum_{i=1}^{m} \mathcal{Q}_{n_{i}}^{i}(f)\right)^{2} \leq \sum_{i=1}^{m} \frac{a_{i}^{2}}{n_{i}} \leq \frac{1}{k_{L}}\left(\sum_{i=1}^{m} a_{i}\right)^{2} .
$$

Thus if $k_{L} \rightarrow \infty$, then the error on $[0, M]$ decreases as $k_{L}^{-1 / 2}$. Furthermore, by (6) the asymptotic constant is smaller than but, for large $M$ and $m$, arbitrarily close to $\int_{\mathbb{R}^{d}}\|x\|^{\beta} \rho(\mathbf{x}) d \mathbf{x}$.

For the error on $[M, \infty)$, we let

$$
\mathcal{I}=\left\{i \geq m+1: n_{i}=0\right\}
$$

Then, for $i, j \in \mathcal{I}$ with $i \leq j$ we have

$$
\begin{aligned}
& \mathbb{E}\left(\int_{P_{i}} f(\mathbf{x}) \rho(\mathbf{x}) d \mathbf{x}\right)\left(\int_{P_{j}} f(\mathbf{y}) \rho(\mathbf{y}) d \mathbf{y}\right) \\
& \quad=\int_{P_{i}} \int_{P_{j}} K(\mathbf{x}, \mathbf{y}) \rho(\mathbf{x}) \rho(\mathbf{y}) d \mathbf{x} d \mathbf{y} \\
& \quad \leq M^{2 \beta} \cdot \operatorname{Vol}\left(P_{i}\right) \cdot \operatorname{Vol}\left(P_{j}\right) \cdot 2^{2 \beta i} \cdot \omega\left(r_{i-1}\right) \cdot \omega\left(r_{j-1}\right) \\
& \quad=a_{i} \cdot a_{j} \cdot 2^{-\beta|j-i|}
\end{aligned}
$$

where we used the inequality

$$
K(\mathbf{x}, \mathbf{y}) \leq \min \left(\|\mathbf{x}\|^{2 \beta},\|\mathbf{y}\|^{2 \beta}\right) .
$$

As in [14] (the proof of Theorem 4) we can show that

$$
\sum_{i, j \in \mathcal{I}} a_{i} \cdot a_{j} \cdot 2^{-\beta|j-i|} \leq \frac{2^{\beta}-1}{2^{\beta}+1} \cdot \sum_{i \in \mathcal{I}} a_{i}^{2} .
$$


Observe that $\operatorname{Int}_{\rho}^{i}(f)-\mathcal{Q}_{n_{i}}^{i}(f)$ and $\operatorname{Int}_{\rho}^{j}(f)-\mathcal{Q}_{n_{j}}^{i}(f)$ are zero mean and pairwise independent whenever $i \neq j$ and at least one of the indices is not in $\mathcal{I}$. Hence,

$$
\begin{aligned}
& \mathbb{E}\left(\int_{\|x\|>M} f(\mathbf{x}) \rho(\mathbf{x}) d \mathbf{x}-\sum_{i=m+1}^{\infty} \mathcal{Q}_{n_{i}}^{i}(f)\right)^{2} \\
& \quad \leq \mathbb{E}\left(\sum_{i \notin \mathcal{I}} \operatorname{Int}_{\rho}^{i}(f)-\mathcal{Q}_{n_{i}}^{i}(f)\right)^{2}+\mathbb{E}\left(\sum_{i \in \mathcal{I}} \operatorname{Int}_{\rho}^{i}(f)\right)^{2} \\
& \leq \frac{2^{\beta}+1}{2^{\beta}-1} \cdot \sum_{i=m+1}^{\infty} \frac{a_{i}^{2}}{\max \left(1, n_{i}\right)} \\
& \leq \frac{2^{\beta}+1}{2^{\beta}-1} \cdot\left(\frac{1}{k_{R}}\left(\sum_{i=m+1}^{m+k_{R}} a_{i}\right)^{2}+\sum_{i=m+k_{R}+1}^{\infty} a_{i}^{2}\right)
\end{aligned}
$$

where we also used (9). The latter sum can be estimated as follows. By monotonicity of $a_{i}$, for every $i \geq m+1$ we have $(i-m) a_{i} \leq \sum_{j=m+1}^{i} a_{j}$. This implies

$$
a_{i}^{2} \leq(i-m)^{-2} \cdot S_{m} \quad \text { with } S_{m}=\left(\sum_{j=m+1}^{\infty} a_{j}\right)^{2}
$$

and

$$
\sum_{i=m+k_{R}+1}^{\infty} a_{i}^{2} \leq k_{R}^{-1} \cdot S_{m} .
$$

(Actually, $\sum_{i=m+k_{R}+1}^{\infty} a_{i}^{2}=o\left(k_{R}^{-1}\right)$ as $k_{R} \rightarrow \infty$.) This and (7) show that if $k_{R} \rightarrow$ $\infty$, then the error on $[M, \infty)$ decreases as $k_{R}^{-1 / 2}$, with the asymptotic constant arbitrarily close, for large $M$, to zero.

The rest of the proof goes as in the proof of Theorem 4 of 14. That is, we increase the parameters $M, m, k_{L}, k_{R}$ and combine them in such a way that for the resulting algorithm $\mathcal{Q}_{n}^{*}$ the error on $[0, M]$ dominates the error on $[M, \infty)$, and $\operatorname{error}\left(\mathcal{Q}_{n}^{*}\right)=C_{n} n^{-1 / 2}$ with $\lim _{n \rightarrow \infty} C_{n}=\int_{\mathbb{R}^{d}} K^{1 / 2}(\mathbf{x}, \mathbf{x}) \rho(\mathbf{x}) d \mathbf{x}$.

One of the possible specific choices of the parameters is discussed in the next section.

\section{Numerical RESUlts}

We first test our algorithm for weight functions

$$
\rho_{1}(\mathbf{x})=\exp \left(-\|\mathbf{x}\|^{2}\right)
$$

and

$$
\rho_{2}(\mathbf{x})=\frac{1-\|\mathbf{x}\|}{1-\|\mathbf{x}\|^{d+3}} .
$$

We use the following strategy in choosing the parameters $M, k_{L}, k_{R}$, and $m$ for given input $n$. We first let

$$
M:=\left\lceil\log _{b}(n)\right\rceil,
$$

where $b=e$ for $\rho_{1}$ and $b=1.05$ for $\rho_{2}$. We have two quantities

$$
S_{1}=\int_{\|\mathbf{x}\| \leq M}\|\mathbf{x}\|^{1 / 2} \rho(\mathbf{x}) d \mathbf{x}
$$


and

$$
S_{2}=\int_{\|\mathbf{x}\| \geq M}\|\mathbf{x}\|^{1 / 2} \rho(\mathbf{x}) d \mathbf{x}
$$

which can be easily computed using one dimensional integrals. Then we choose

$$
k_{L}:=n \cdot\left\lceil\frac{\sqrt{S_{1}}}{\sqrt{S_{1}}+\sqrt{S_{2}}}\right\rceil
$$

and

$$
k_{R}:=n-k_{L} .
$$

Finally,

$$
m:=\left\lceil k_{L}^{z}\right\rceil,
$$

where $z=0.9$. The sample size of the algorithm is computed on the fly.

To generate points from rings, we first generate points from the uniform distribution on the unit sphere and then generate their radii. To this end, we use methods from $[3,[5]$. Both steps above require a uniform generator from $[0,1]$. We mainly use:

- the generator of M. Matsumoto and T. Nishimura (see [10]);

- the maximally equidistributed combined Tausworthe generator by P. L'Ecuyer (see [4]);

- the generator of R. M. Ziff (see [16]).

Implementations of these and many other generators can be found in the GNU Scientific Library (GSL) 2

The functions to be integrated for the Gaussian weight $\rho_{1}$ are

$$
f_{1}(\mathbf{x}):=\cos (|| \mathbf{x}||)
$$

and

$$
f_{2}\left(x_{1}, \ldots, x_{d}\right)=\sum_{k=1}^{d} \frac{1}{1+\sqrt{\left|x_{k}\right|}} .
$$

For the rational weight $\rho_{2}$, we test the following function

$$
f_{3}\left(x_{1}, \ldots, x_{d}\right)=\sum_{k=1}^{d}\left|x_{k}\right|
$$

The computations are done for dimensions $d=10,25$ and, additionally, for Gaussian weight in $d=100$. The results are shown in Figures 1 through 5 . These are plots of functions of relative errors of sample sizes.

The function $f_{1}$ was discussed in [1], where the algorithm QMC-GF, based on the generalized Faure sequence $(\underline{15})$, was used to compute the integrals. We point out that $f_{1}$ creates essentially one dimensional cases, and our test result confirms this. Moreover, our algorithm gives the error several times smaller than QMC-GF.

It is interesting to note that for $f_{2}$, higher dimensional cases generally result in smaller errors; see Figures 3 and 4.

We also provide results for valuation of the mortgage-backed securities; see, e.g., Paskov [12. In this case, we have the normalized Gaussian weight

$$
\rho_{3}(\mathbf{x})=(2 \pi)^{-d / 2} \exp \left(-\|\mathbf{x}\|^{2} / 2\right),
$$

\footnotetext{
${ }^{2}$ See http://sources.redhat.com/gsl/
} 


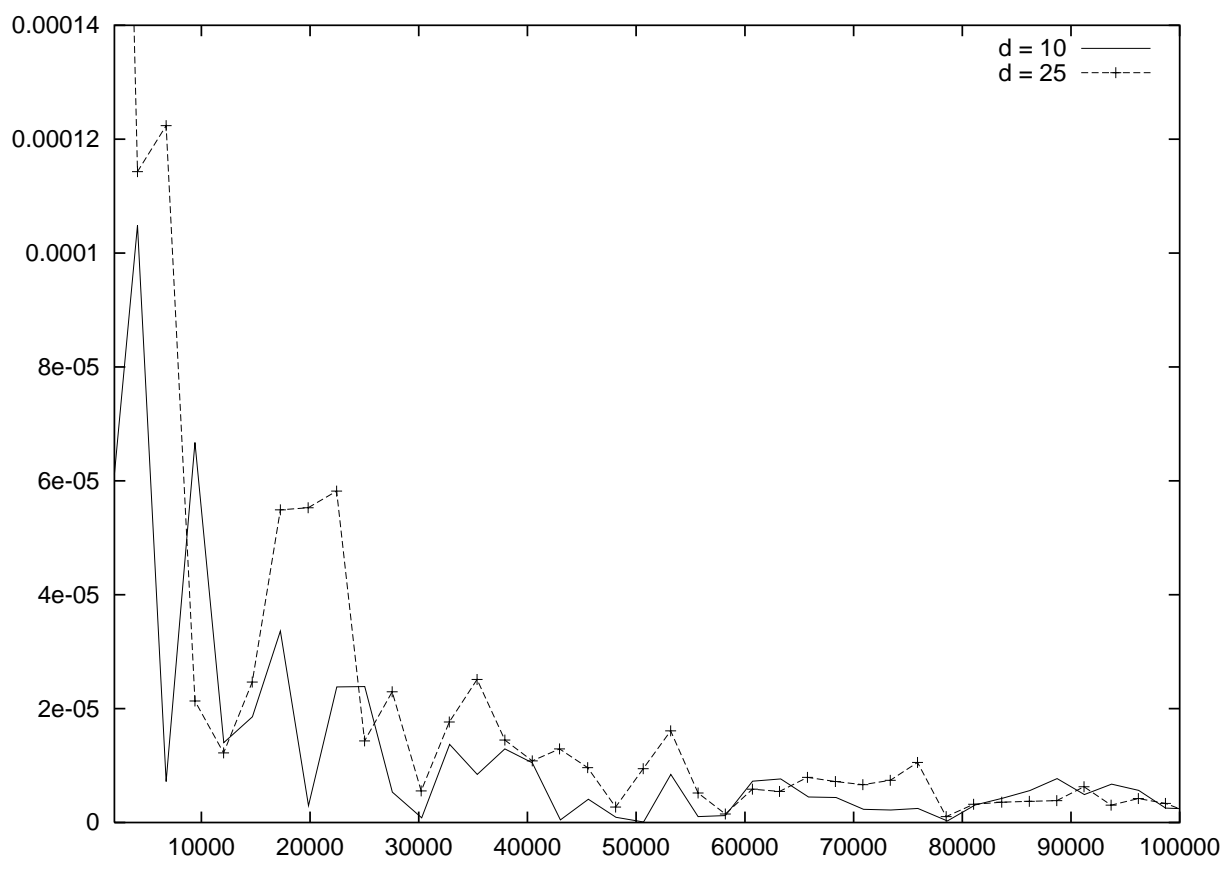

Figure 1. $f_{1}(\mathbf{x}), d=10$ and 25 , Gaussian weight

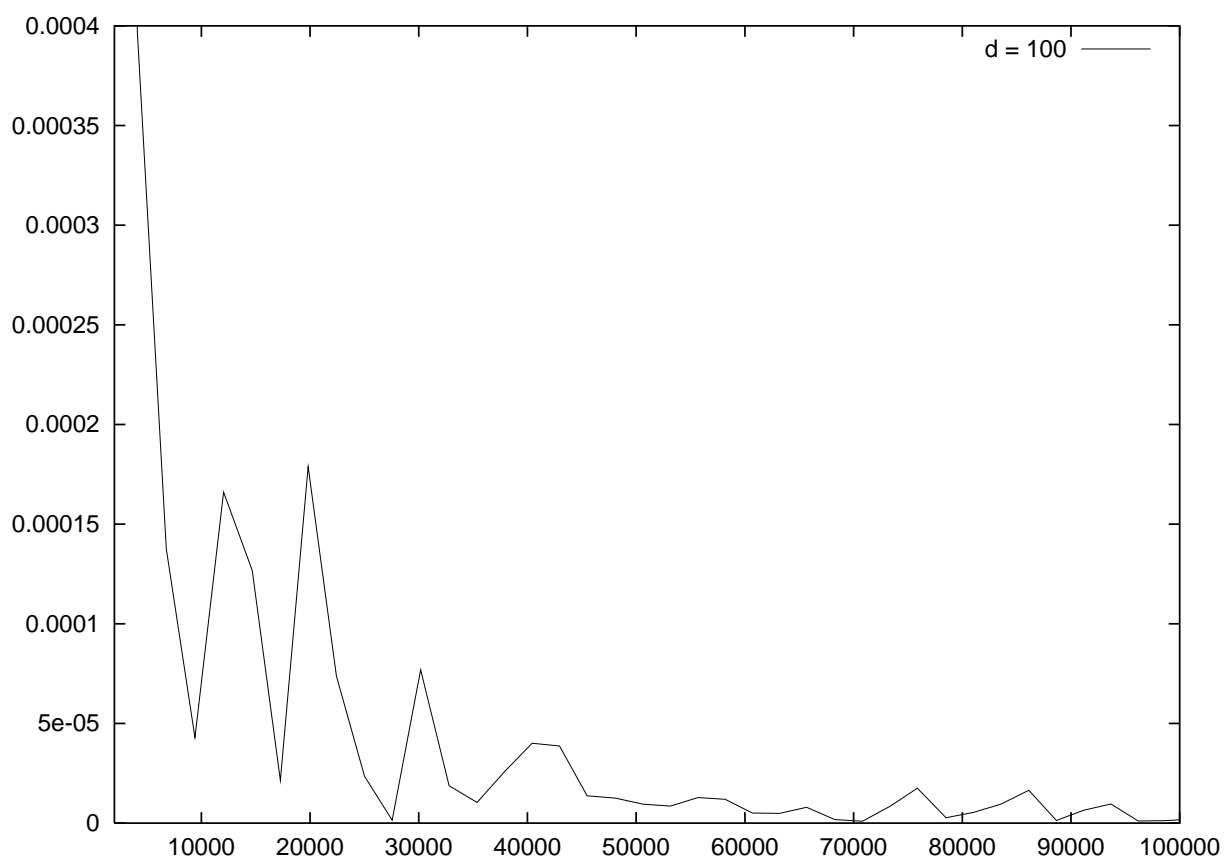

Figure 2. $f_{1}(\mathbf{x}), d=100$, Gaussian weight 


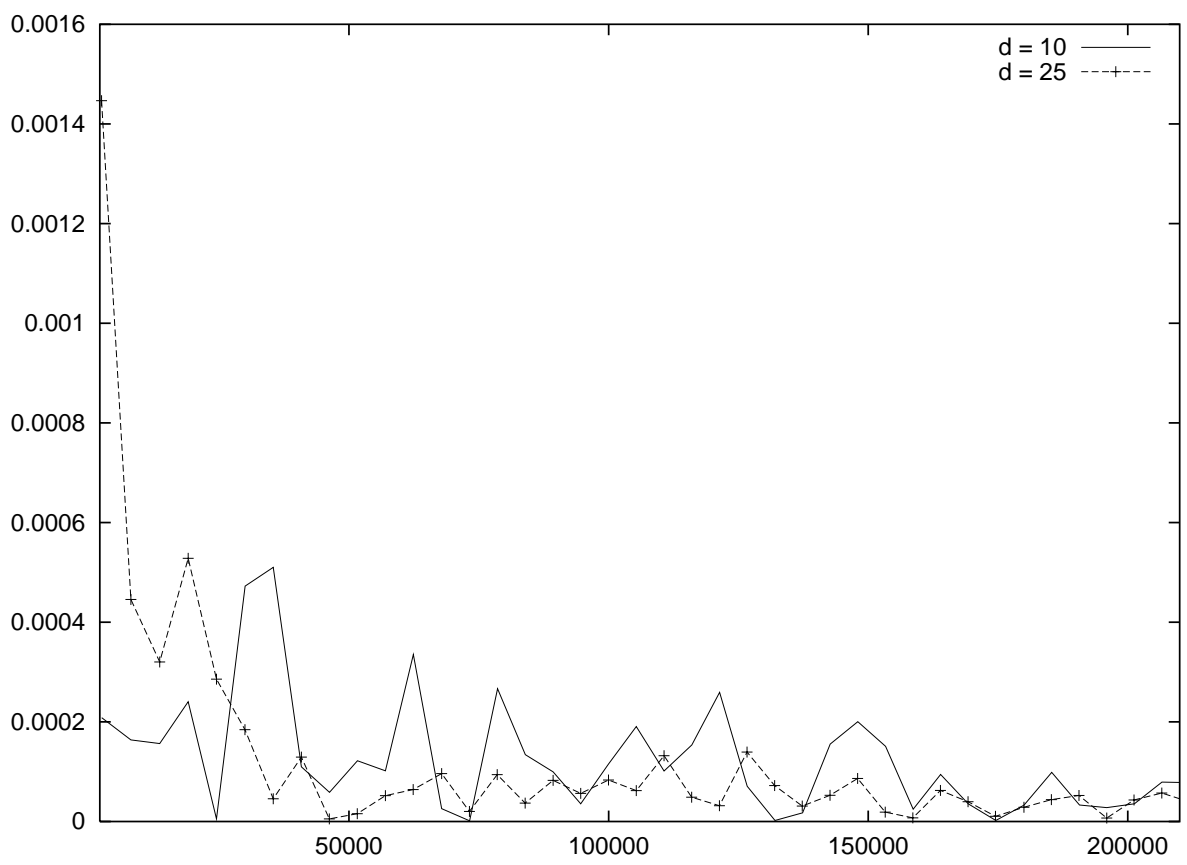

Figure $3 . f_{2}(\mathbf{x}), d=10$ and 25 , Gaussian weight

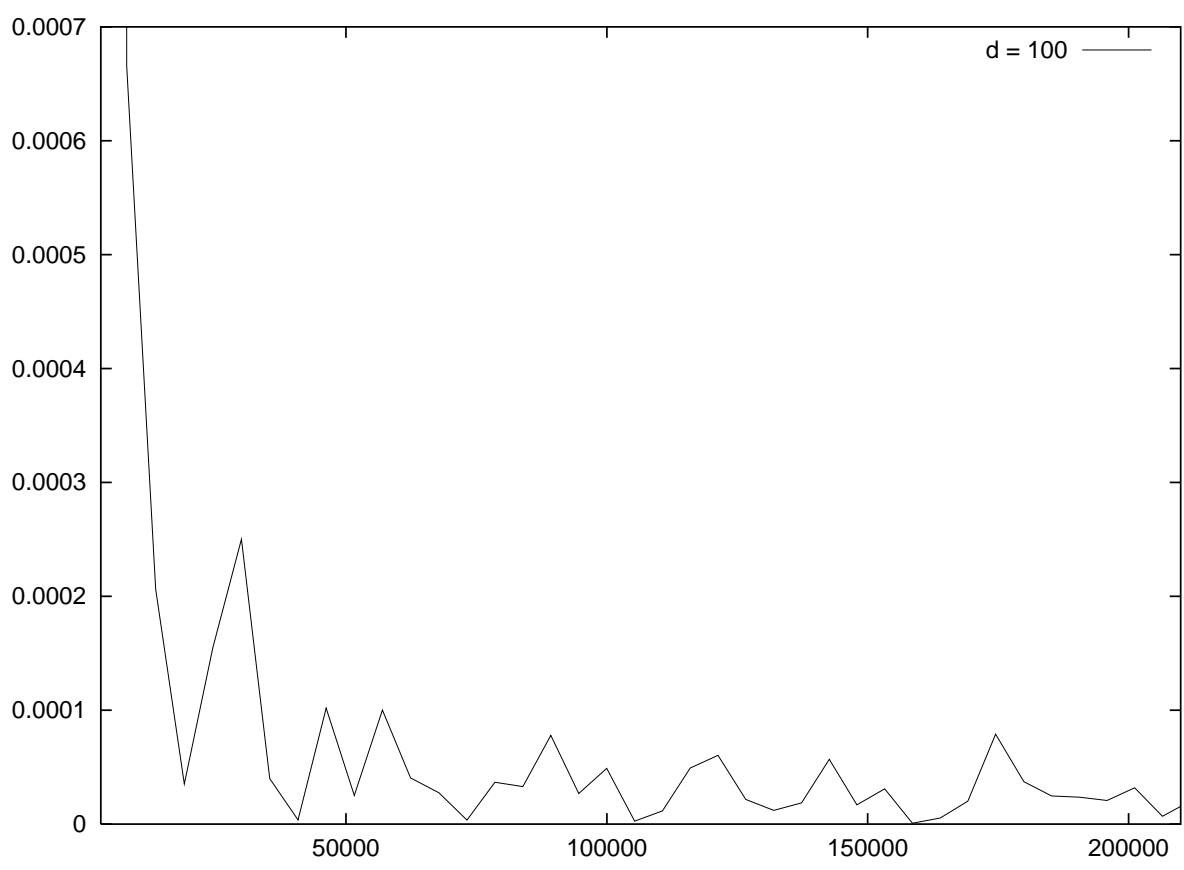

Figure 4. $f_{2}(\mathbf{x}), d=100$, Gaussian weight 


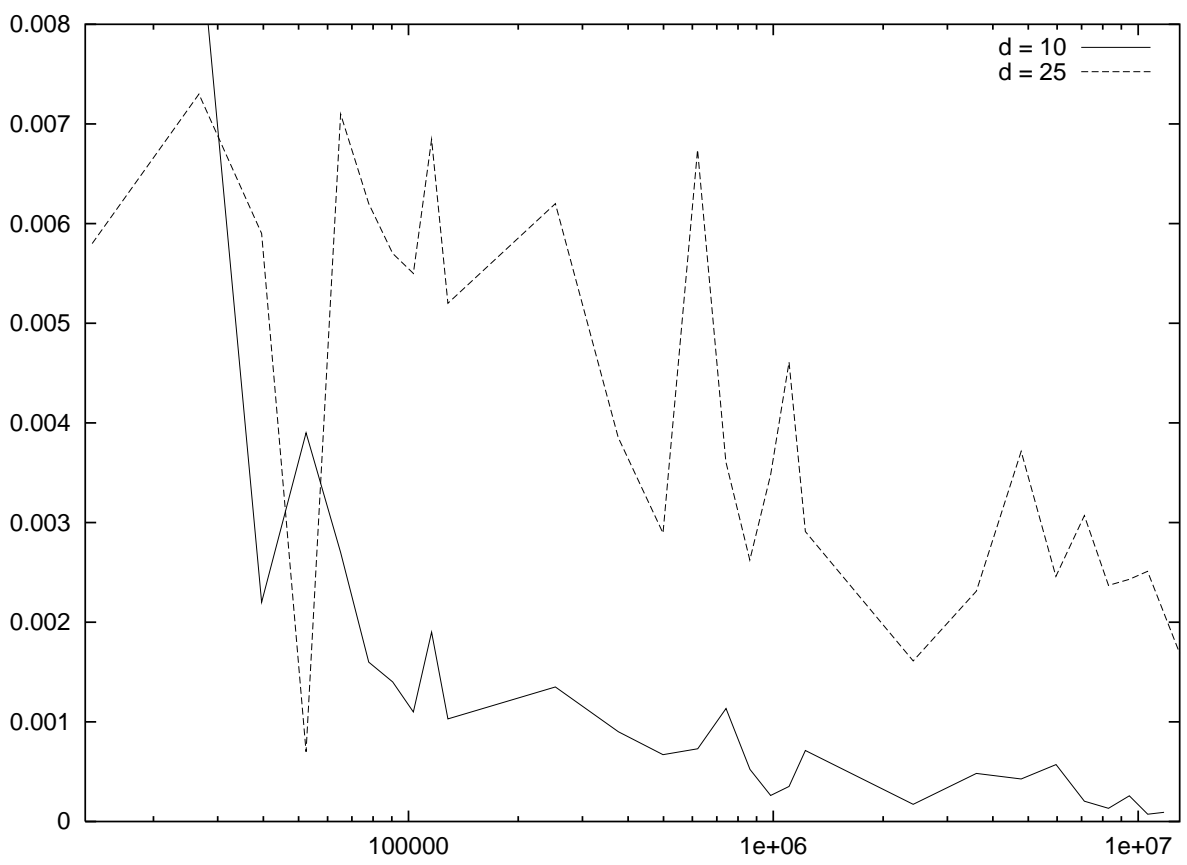

Figure 5. $f_{3}(\mathbf{x}), d=10$ and 25 , rational weight

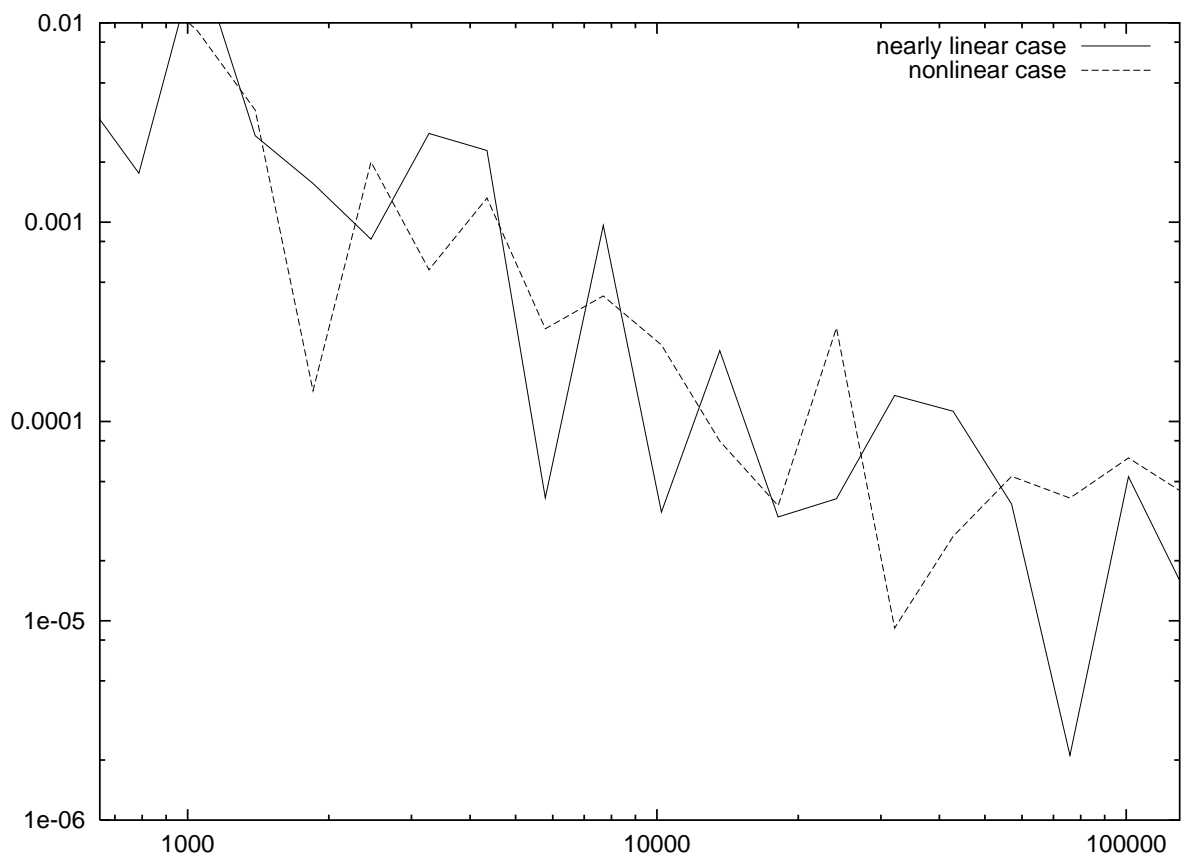

FiguRE 6. Mortgage-backed securities, $d=360$ 
and the dimension is $d=360$. We choose $M=25$ with the other parameters as for $\rho_{1}$ and $\rho_{2}$. Paskov [12] was the first to notice, for this particular problem, an advantage of quasi-Monte Carlo computation over straightforward random Monte Carlo. His numerical results were then improved by more sophisticated methods; see [1, 2, 6, 11]. As in the tests from those papers, we consider nearly linear and nonlinear cases. Results are shown in Figure 6. Observe that the convergence of our algorithm is much better than $n^{-1 / 2}$ and that the errors are comparable to that obtained by Paskov for quasi-Monte Carlo. Methods specially designed for this particular integrands/weights, like those of Caflisch and Morokoff [1] or Genz [6], perform better 3 , however, not much. Indeed, we lose, more or less, 1.5 decimal digits in the nearly linear case and 1 decimal digit in the nonlinear case. It is also noticeable that our algorithm seems not to distinguish between the nearly linear and nonlinear cases, although the nonlinear case was meant to be more difficult. This agrees with the theoretical property that the algorithm is not sensitive to an increased smoothness of the integrand (only Hölder continuity counts).

\section{ACKNOWLEDGMENTS}

The authors thank Ronald Cools, Harald Niederreiter, and the anonymous referee for valuable comments and suggestions on the paper.

P. Gajda and L. Plaskota were partially supported by the State Committee for Scientific Research of Poland under Grant 5 P03A 007 21. Y. Li and G. W. Wasilkowski were partially supported by the National Science Foundation under Grant CCR-0095709.

\section{REFERENCES}

1. R.E. Caflisch and W. Morokoff, Quasi-Monte Carlo computation of a finance problem, in K.T. Fang and F.J. Hickernell, eds., Workshop on Quasi-Monte Carlo Methods and Their Applications, 1996, pp.15-30.

2. R.E. Caflisch, W. Morokoff and A.B. Owen, Valuation of mortgage backed securities using Brownian bridges to reduce effective dimension, J. Comp. Finance, 1996, 1, pp.27-46.

3. L. Devroye, "Non-Uniform Random Variate Generation", Springer, New York, 1986. MR 87i:65012

4. P. L'Ecuyer, Maximally Equidistributed Combined Tausworthe Generators, Mathematics of Computation, 65 (1996) pp.203-213. MR 96d:65017

5. G.S. Fishman, "Monte Carlo: Concepts, Algorithms, and Applications", Springer, New York, 1996. MR 97g:65017

6. A. Genz, Stochastic methods for multiple integrals over unbounded regions, Mathematics and computers in simulation, 47 (1998), pp.287-298 MR 99d:65014

7. S. Haber, A modified Monte-Carlo quadrature, I, Math. Comp. 20 (1966), pp.361-368. MR 35:1178

8. S. Haber, A modified Monte-Carlo quadrature, II, Math. Comp 21 (1967), pp.388-397. MR 38:2922

9. P. Mathe and G. Wei, Quasi-Monte Carlo integration over $\mathbb{R}^{d}$, to appear.

10. M. Matsumoto and T. Nishimura, Mersenne Twister: a 623-dimensionally equidistributed uniform pseudorandom number generator, ACM Transactions on Modeling and Computer Simulation, 1 (1998), pp.3-30.

11. A. Papageorgiou and J.F. Traub, Faster Evaluation of Multidimensional Integrals, Computers in Physics, Nov./Dec. 1997, pp.574-578.

12. S.H. Paskov, New methodologies for valuing derivatives, in S. Pliska and M. Dempster, eds., Mathematics of Derivative Securities, Issac Newton Inst., Cambridge Univ. Press, 1996. MR 98k:90014

\footnotetext{
${ }^{3}$ In terms of errors with the same sample size.
} 
13. L. Plaskota, K. Ritter, and G.W. Wasilkowski, Average case complexity of weighted integration and approximation over $\mathbb{R}^{d}$ with isotropic weight, in K.T. Fang, J.F. Hickernell, and H. Niederreiter, eds., Monte Carlo and Quasi Monte Carlo 2000, Hong-Kong, Springer 2002, pp.446-459.

14. L. Plaskota, K. Ritter, and G.W. Wasilkowski, Optimal designs for weighted approximation and integration of stochastic processes on $[0, \infty), 2002$, submitted.

15. S. Tezuka, "Uniform Random Numbers: Theory and Practice," Kluwer Academic Publishers, Boston, 1995.

16. R. M. Ziff, Four-tap shift-register-sequence random-number generators, Computers in Physics, Jul/Aug 1998, pp.385-392.

Department of Mathematics, Informatics, and Mechanics, Warsaw University, Ul. Banacha 2, 02-097 Warsaw, Poland

E-mail address: piotrg@mimuw.edu.pl

Mathematics and Computer Science Department, Georgia Southern University, 0203 Georgia Avenue, Statesboro, Georgia 30460-8093

E-mail address: yming@gasou.edu

Department of Mathematics, Informatics, and Mechanics, Warsaw University, ul. Banacha 2, 02-097 Warsaw, Poland

E-mail address: leszekp@mimuw.edu.pl

Department of Computer Science, University of Kentucky, 773 Anderson Hall, LexINGTON, KENTUCKY 40506-0046

E-mail address: greg@cs.uky.edu 